\title{
Intravenous glutathione for skin lightening: Inadequate safety data
}

\author{
L M Davids, ${ }^{1} \mathrm{PhD} ; \mathbf{J}$ C van Wyk, ${ }^{2} \mathrm{PhD} ; \mathbf{N}$ P Khumalo, ${ }^{2} \mathrm{MB} \mathrm{ChB}, \mathrm{FCDerm}, \mathrm{PhD}$ \\ ${ }^{1}$ Department of Human Biology, Faculty of Health Sciences, University of Cape Town and Groote Schuur Hospital, Cape Town, South Africa \\ ${ }^{2}$ Division of Dermatology, Faculty of Health Sciences, University of Cape Town and Groote Schuur Hospital, Cape Town, South Africa
}

Corresponding author: N P Khumalo (n.khumalo@ uct.ac.za)

\begin{abstract}
Background. Glutathione (GSH) is the most abundant naturally occurring non-protein thiol that protects mammalian cells from oxidative stress. Intravenous (IV) GSH for skin lightening is advertised by clinics in South Africa and internationally online, yet to date no published review on the subject exists.

Methods. We conducted a MEDLINE search (to 30 September 2015) of GSH use for skin lightening and of all indications in medicine, to evaluate its safety.

Results. Two controlled clinical trials (GSH capsules: 60 patients; $2 \%$ glutathione disulphide lotion: 30 patients) and a case series (GSH lozenges: 30 patients) reported a significantly decreased melanin index. A case series (GSH soap: 15 patients) reported skin lightening based on photography. Two systematic reviews of IV GSH for preventing chemo-induced toxicity and a third review of adjuvant therapy for Parkinson's disease altogether included 10 trials. Most trials reported either no or minimal GSH adverse effects, but all had treatment durations of a few doses (IV) or 4 - 12 weeks. No study reported long-term IV GSH use.

Conclusion. In spite of widespread reported use, there are no studies of IV GSH use for skin lightening or of its safety for chronic use (for any indication). The switch from brown to red melanin production may increase the risk of sun-induced skin cancers in previously protected individuals. Regulatory assessment of systemic GSH administration for cosmetic use by the Medicines Control Council seems urgently warranted to protect consumers from potential side-effects and from complications of IV infusions. This is especially concerning because of reports of GSH bought online. Effective topical GSH may be useful for hyperpigmented skin disorders, but this requires scientific scrutiny. The debate on the merits of cosmetic skin lightening is best handled by multidisciplinary teams.
\end{abstract}

S Afr Med J 2016;106(8):782-786. DOI:10.7196/SAMJ.2016.v106i8.10878

Dermatologists use depigmenting creams with various active ingredients in the treatment of melasma and post-inflammatory hyperpigmentation. ${ }^{[1]}$ Skin lightening or bleaching refers to the cosmetic practice of applying depigmenting agents not as treatment for hyperpigmentation but with the deliberate aim of achieving a lighter skin colour. It is a practice that is common in many places (e.g. India, Africa and America) with pigmented populations and a history of improved social status with lighter complexion. Adverse effects are associated with active ingredients (mercury, hydroquinone and potent steroids) in depigmenting creams and are illegal in cosmetics in many countries. Further illegal ingredients in Africa have been shown to be imported from Europe (in spite of a European Union ban). ${ }^{[2]}$

Glutathione (GSH) was first discovered by Hopkins ${ }^{[3]}$ in 1921 in yeasts, and subsequently in other tissues. ${ }^{[4]}$ It is a tripeptide composed of L-cysteine, glycine and glutamate that is synthesised intracellularly. ${ }^{[5,6]}$ It is considered the main redox buffer in human cells owing to its large amount of reducing equivalents, ${ }^{[7]}$ and is an important enzyme cofactor that serves as a neuromodulator in the central nervous system. The tripeptide exists intracellularly either in an oxidised glutathione disulphide (GSSG) or reduced state (GSH), and maintaining an optimal GSH:GSSG ratio in the cell is critical for prevention of oxidative damage and for cell survival (Fig. 1). ${ }^{\left[{ }^{[3}\right.}$ An imbalance in GSH and its use as a marker of oxidative stress is reported in many diseases including cancer, neurodegenerative disorders, cystic fibrosis, HIV, ${ }^{[9]}$ diabetes mellitus, ${ }^{[10]}$ anorexia nervosa $^{[11]}$ and autism ${ }^{[12,13]}$ and in low-birth-weight neonates. ${ }^{[14]}$

Reports of systemic skin lightening with GSH have appeared with increasing frequency on social media, and clinics advertise it online in many countries (Africa, the USA, Canada, Mexico, etc.). Our objective was to conduct a literature search to identify all academic reports of GSH use for skin lightening and all clinical trials of GSH use for all indications in medicine.

\section{Methods}

Two MEDLINE searches for studies published up to September 2015 were conducted. The search terms for the first were 'glutathione AND skin lightening', and for the second 'glutathione AND randomised controlled trial'. Inclusion criteria were any treatment report of GSH for skin lightening (or hyperpigmentation) and GSH for any randomised control trial (RCT) for the first and second searches, respectively. Abstracts were read independently by two authors to identify relevant articles.

\section{Results}

Nine articles were identified from the first MEDLINE search. Six publications mentioned GSH as part of reviews related to melanin (kojic acid in rats, ${ }^{[15]}$ piceatannol inhibition of mushroom tyrosinase, ${ }^{[16]}$ oral zinc sulphate murine hair hypopigmentation, ${ }^{[17]}$ hydroquinone occupational exposure ${ }^{[18]}$ (and toxicity for skin lightening) ${ }^{[19]}$ and natural ingredient-containing treatments for hyperpigmentation. ${ }^{[20]}$ A seventh article was an extensive review of biochemical mechanisms of how GSH causes depigmentation in cell cultures and laboratory animals. ${ }^{[2]}$ Five clinical reports (4 published since 2012) of the use of GSH for skin lightening were identified ( 2 from the MEDLINE search and 3 from references). The first, a pharmacokinetic study of GSH in seven participants, did not measure skin lightening 


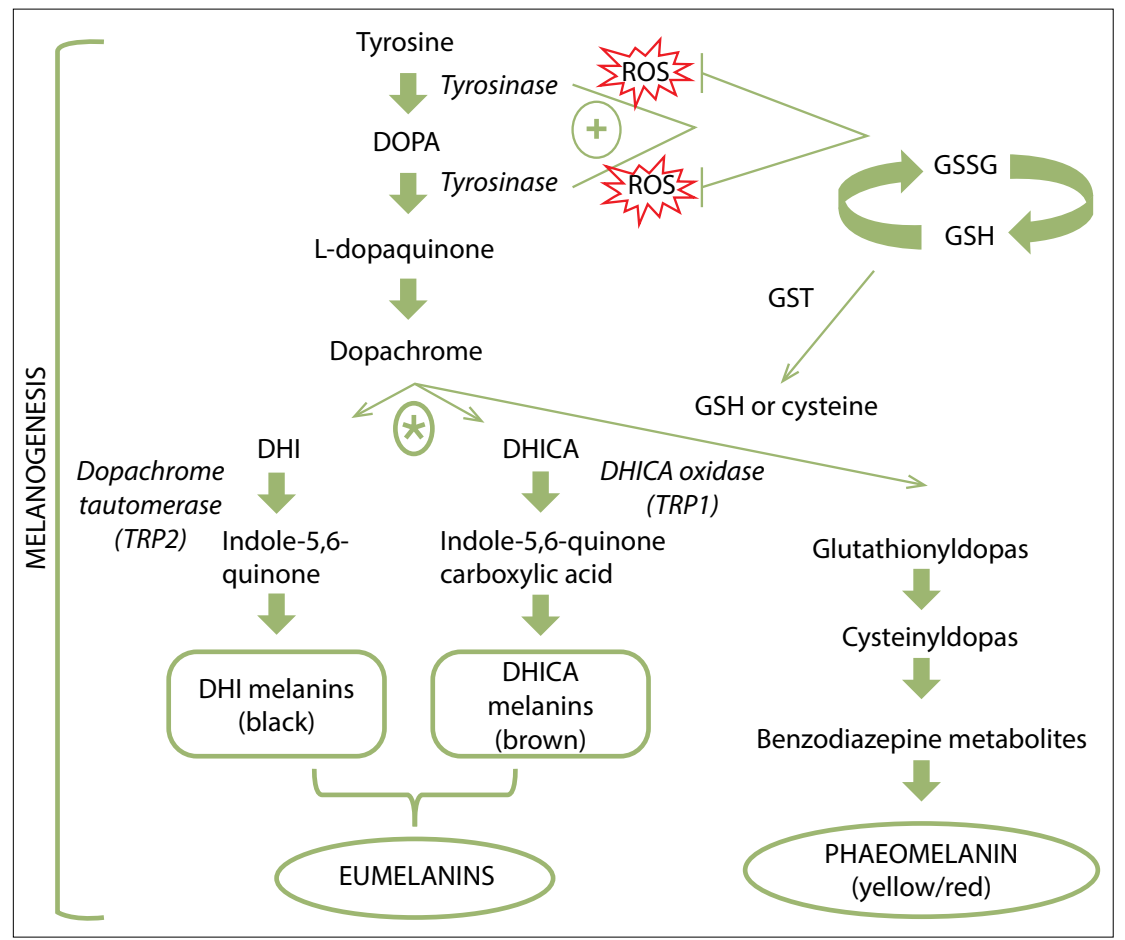

Fig. 1. GSH and its effect on skin lightening. Reactive oxygen species (ROS) have a direct activation effect on tyrosinase. Reduced GSH neutralises ROS formation and thus indirectly inhibits tyrosinase. At the dopachrome step ${ }^{*}$ of the melanogenic pathway, interaction of thiols such as reduced GSH and cysteine bind with dopaquinone to produce thioldopas and favour phaeomelanogenesis. GST catalyses binding of GSH and dopaquinone. $($ DOPA $=$ dihydroxyphenylalanine; $D H I=$ dihydroxyindole; DHICA = dihydroxyindole carboxylic acid.)

or report side-effects. ${ }^{[22]} \mathrm{A}$ case series of 15 patients from India treated with a GSHcontaining soap for melanosis of the face reported lightening (in 11/15 after 3 months) based on clinical photographs. However, no mention was made of how the conditions for photography were standardised or of followup on stopping treatment. ${ }^{[23]}$ A controlled trial from Thailand tested $250 \mathrm{mg}$ GSH capsules twice daily in two groups each of 30 medical students over 4 weeks. They reported a decreased melanin index measured at six body sites (but statistically significant only at two sites, namely the right side of the face $(p=0.021)$ and the sun-exposed left forearm $(p=0.036)) .{ }^{[24]}$ Watanabe et al. ${ }^{[25]}$ from Japan reported a significant reduction in the melanin index (mean (standard deviation), week 0: 272.77 (26.17); week 10: 243.47 (26.31)) in subjects treated with a GSSG-containing lotion measured with a Mexameter MX18 (Courage + Khazaka Electronic $\mathrm{GmbH}$, Germany). Investigators and participants also subjectively scored GSSGtreated skin as lighter. The most recent study is an uncontrolled trial of 30 Filipino women. The authors reported a significant reduction in melanin index (measured with a portable mexameter $)(p<0.0001)$ after $500 \mathrm{mg}$ GSH lozenges were administered daily for 8 weeks. ${ }^{[2]}$ None of the studies reported significant adverse effects or followup beyond the study period (Table 1).

Of the plethora of articles retrieved from the second MEDLINE search, we identified 28 systematic reviews of animal studies, ${ }^{[39-41]}$ GSH-related genetic polymorphisms linked to various cancers (colorectal cancer, ${ }^{[42]}$ leukaemia, ${ }^{[43,44]}$ lung cancer, ${ }^{[45,46]}$ bladder cancer, ${ }^{[47]}$ gastric cancer, ${ }^{[48,49]}$ prostate cancer, ${ }^{[50-53]}$ adult brain tumours, ${ }^{[54]}$ basal cell carcinoma $^{[55]}$ ) and linked to other disorders (autism, ${ }^{[12,13]}$ hypertension, ${ }^{[56,57]}$ respiratory diseases, ${ }^{[58,59]}$ cataract, ${ }^{[60]}$ myelodysplastic syndrome, ${ }^{[61,62]}$ glioma $^{[63]}$ and male idiopathic infertility. ${ }^{[64]}$ ) There were 9 RCTs identified from two systematic reviews of the use of GSH to reduce chemotherapyinduced toxicity (6 cisplatin, 2 axaliplatin, 1 platinum); most suggested less toxicity in GSH groups. A systematic review of GSH as an adjuvant therapy in Parkinson's disease identified one controlled trial ${ }^{[65]}$ showing doubtful benefit. It was noteworthy that most studies did not report adverse events of GSH, and any reported were mild (Table 1).

\section{Discussion}

The idea of GSH-induced hypopigmentation may stem from early studies linking sulfhydral-containing compounds to the inhibition of melanogenesis or from early anecdotal observations in Parkinson's disease. Proposed mechanisms of its action include inactivation of the melanogenic enzyme, tyrosinase, influencing the switch from eumelanin to phaeomelanin. ${ }^{[66,67]}$ During melanogenesis, tyrosinase is responsible for the conversion of L-tyrosine to L-DOPA and subsequently to dopaquinone, then the pathway bifurcates to produce eumelanin or phaeomelanin. At a critical point in the melanogenic pathway (asterisk, Fig. 1), thiols (cysteine and GSH) can react with L-dopaquinone to produce glutathionyldopa, or act as a reservoir of L-cysteine by conjugating with L-dopaquinone to produce cysteinyldopa. These two thioldopa substrates serve as a precursor to enhance the switch from eumelanogenesis to phaeomelanogenesis, resulting in lighter skin pigmentation. ${ }^{[68,69]}$ This effect of GSH on skin pigmentation was reported half a century ago, with black human skin shown to exhibit lower levels of GSH than white skin. ${ }^{[70]}$ In addition, GSH can act to lighten the skin directly through the quenching of free radicals and peroxides that have been shown to induce tyrosinase activity. ${ }^{[71]}$ However, more evidence is needed to prove this unequivocally.

GSH therefore has the potential to lighten human skin. However, the only reliable safety data on GSH are of sporadic use during chemotherapy cycles, for a few weeks at most. There are no data on adverse effects of chronic high-dose GSH as used for skin lightening.

All chemotoxicity studies used injectable GSH. Reactive oxygen species are easily decomposed in aqueous solution; this may explain the novelty of drug delivery as lozenges, which may be more stable (although two participants complained about the taste). The oral route reduces potential adverse events associated with intravenous (IV) administration but is associated with low bioavailability. Effective topical GSH ${ }^{[25]}$ may be useful for dermatologists treating hyperpigmentation, but it is worth noting that GSH as a thiol interacts with metalloid complexes that render it ineffective. Patients should be advised to avoid using GSH with over-the-counter skin lightening creams that may contain mercury. ${ }^{[1]}$

All identified published trials report mild or no side-effects of GSH use. However, study duration was a maximum of 12 weeks. We identified one case series that reported intolerable adverse effects leading to discontinuation of $5 \mathrm{mg}$ oral GSH daily as adjuvant treatment for hepatocellular carcinoma (HCC). Seven of 8 patients died within 
1 year. However, the very severe prognosis associated with HCC was a likely confounder. ${ }^{[72]}$ The effect of long-term administration of high doses of GSH on cells or organ systems remains unclear. Further- more, since GSH causes a switch from eumelanin to phaeomelanin it may increase UV photosensitivity, DNA damage and skin cancers in previously protected populations. ${ }^{[73]}$ Of major concern are

Table 1. Current list of human clinical trials associated with GSH

\begin{tabular}{|c|c|c|c|c|}
\hline Reference & $\begin{array}{l}\text { Country of origin } \\
\text { Study type } \\
\text { Indication }\end{array}$ & $\begin{array}{l}\text { Subjects, } n \text { (sex), age } \\
\text { GSH dose and duration } \\
\text { Study duration }\end{array}$ & Outcomes & Adverse events \\
\hline \multicolumn{5}{|l|}{ Skin whitening } \\
\hline $\begin{array}{l}\text { Hong et al.. }{ }^{[22]} \\
2005\end{array}$ & $\begin{array}{l}\text { Korea } \\
\text { Cases (uncontrolled) } \\
\text { Pharmacokinetic study }\end{array}$ & $\begin{array}{l}7 \text { (male), } 22-23 \mathrm{yr} \\
65.5(\mathrm{SD} 4.5) \mathrm{kg}, 50 \mathrm{mg} \\
\text { GSH } / \mathrm{kg} \text { body weight IV } \\
\text { over } 10 \mathrm{~min}, 10 \mathrm{~d}\end{array}$ & $\begin{array}{l}\text { IV GSH oxidised to GSSG (half-life }= \\
10 \mathrm{~min} \text { ) } \\
\text { Loading dose }=1.69 \mathrm{~g} / \mathrm{kg} \\
\text { Maintenance dose }=5.70 \mathrm{~g} / \mathrm{h} / \mathrm{kg} \text { to } \\
\text { reach extracellular concentration } \\
\text { required to suppress intracellular ROS }\end{array}$ & None reported \\
\hline $\begin{array}{l}\text { Arjinpathana and } \\
\text { Asawonda, }{ }^{[2]]} 2012\end{array}$ & $\begin{array}{l}\text { Bangkok, Thailand } \\
\text { RCT (double-blind, } \\
\text { placebo) } \\
\text { Skin whitening }\end{array}$ & $\begin{array}{l}60 \text { ( } 18 \text { male, } 42 \text { female), } \\
19 \text { - } 22 \mathrm{yr} \\
250 \mathrm{mg} \text { capsules GSH twice } \\
\text { daily, } 4 \mathrm{wk}\end{array}$ & $\begin{array}{l}\text { Significant reduction in melanin indices } \\
\text { (UV spots) as measured by VISIA } \\
\text { (Canfield Scientific Inc., USA) at all six sites } \\
\text { in subjects who received GSH v. controls }\end{array}$ & Flatulence \\
\hline $\begin{array}{l}\text { Sriharsha et al.., }{ }^{[23]} \\
2015\end{array}$ & $\begin{array}{l}\text { India } \\
\text { Pilot study } \\
\text { GSH soap: melanosis of } \\
\text { the face }\end{array}$ & $\begin{array}{l}15,15-70 \mathrm{yr} \\
3 \mathrm{mo}\end{array}$ & $\begin{array}{l}\text { Decreased hyperpigmentation in } 11 / 15 \\
\text { patients after } 3 \text { mo }\end{array}$ & None reported \\
\hline $\begin{array}{l}\text { Watanabe et al. },^{[25]} \\
2014\end{array}$ & $\begin{array}{l}\text { Ibaraki, Japan } \\
\text { RCT (double-blind, placebo) } \\
\text { Skin whitening }\end{array}$ & $\begin{array}{l}30 \text { (female), } 30 \text { - } 50 \mathrm{yr} \\
2 \% \text { GSSG lotion twice daily, } \\
10 \mathrm{wk}\end{array}$ & $\begin{array}{l}\text { Weeks } 6 \text { and 10: } \\
\text { Melanin index sign lower GSSG v. placebo } \\
\text { Keratin index sign lower GSSG v. placebo }\end{array}$ & $\begin{array}{l}\text { Mild erythema of } \\
\text { the face }(n=1)\end{array}$ \\
\hline $\begin{array}{l}\text { Handog et al., }{ }^{[26]} \\
2015\end{array}$ & $\begin{array}{l}\text { Manilla, Phillipines } \\
\text { Single-arm trial (not } \\
\text { blinded) } \\
\text { GSH-containing lozenges } \\
\text { Skin whitening }\end{array}$ & $\begin{array}{l}30 \text { (female), } 22-42 \mathrm{yr} \\
500 \mathrm{mg} \text { daily, } 8 \mathrm{wk}\end{array}$ & $\begin{array}{l}\text { Decreased melanin index after } 2 \mathrm{wk} \\
\text { All subjects showed a significant } \\
\text { decrease in melanin index from baseline } \\
(p<0.0001)\end{array}$ & $\begin{array}{l}\text { Sore gums }(n=1) \\
\text { Undesirable } \\
\text { flavour/texture of } \\
\text { lozenge }(n=1)\end{array}$ \\
\hline \multicolumn{5}{|c|}{ Chemotherapy drugs neuroprotection } \\
\hline $\begin{array}{l}\text { Cascinu et al. } .^{[27]} \\
1995\end{array}$ & $\begin{array}{l}\text { Italy } \\
\text { RCT (double-blind, placebo) } \\
\text { Prevent cisplatin toxicity } \\
\text { in gastric cancer }\end{array}$ & $\begin{array}{l}50 \\
\text { GSH } 1.5 \mathrm{~g} / \mathrm{m}^{2} \text { in } 100 \mathrm{~mL} \\
\text { saline IV over } 15 \mathrm{~min} \\
600 \mathrm{mg} \text { GSH IM, days } 2-5 \\
15 \mathrm{wk}\end{array}$ & $\begin{array}{l}\text { Neuropathy } \\
\text { Week 9: } 0 \text { GSH v. } 16 \text { placebo } \\
\text { Week 15: 4/24 GSH v. } 16 / 18 \text { placebo }\end{array}$ & None reported \\
\hline $\begin{array}{l}\text { Colombo et al. }{ }^{[28]} \\
1995\end{array}$ & $\begin{array}{l}\text { Italy } \\
\text { Random, phase II } \\
\text { Prevent cisplatin toxicity } \\
\text { in relapsed ovarian cancer }\end{array}$ & $\begin{array}{l}33 \\
50 \mathrm{mg} / \mathrm{m}^{2} \text { weekly } \pm 2.5 \mathrm{~g} / \mathrm{m}^{2} \\
\text { GSH, } 9 \text { wk }\end{array}$ & $\begin{array}{l}\text { Higher ( } 100 \% \text { dose) cisplatin intensity } \\
\text { was received by } 56 \% \text { GSH v. } 27 \% \\
\text { control }\end{array}$ & None reported \\
\hline $\begin{array}{l}\text { Parnis et al.. }{ }^{[29]} \\
1995\end{array}$ & $\begin{array}{l}\text { Australia } \\
\text { RCT (double-blind, } \\
\text { placebo) } \\
\text { Prevent cisplatin toxicity } \\
\text { in ovarian cancer }\end{array}$ & $\begin{array}{l}12 \\
\text { GSH } 1.5 \mathrm{~g} / \mathrm{m}^{2} \text { over } 15 \mathrm{~min} \\
\text { CDDP } 40 \mathrm{mg} / \mathrm{m}^{2} \text { over } 2 \mathrm{~h} \text { for } \\
2,3 \text { or } 4 \text { consecutive days } \\
\mathrm{NR}\end{array}$ & No significant protection & None reported \\
\hline $\begin{array}{l}\text { Bogliun et al., }{ }^{[30]} \\
1992\end{array}$ & $\begin{array}{l}\text { Italy } \\
\text { Placebo controlled } \\
\text { Prevent cisplatin toxicity } \\
\text { in ovarian cancer }\end{array}$ & $\begin{array}{l}33 \\
\mathrm{CDDP} \text { total dose } 500-675 \\
\mathrm{mg} / \mathrm{m}^{2} \pm \mathrm{GSH} 2.5 \mathrm{~g} / \mathrm{m}^{2} \mathrm{IV} \\
\text { over } 15 \mathrm{~min}, 1 \mathrm{wk}\end{array}$ & $\begin{array}{l}\text { Less severe neurotoxicity after } \\
\text { co-treatment with all methods }\end{array}$ & $\begin{array}{l}\text { Similar in both } \\
\text { groups except } \\
\text { oliguria greater in } \\
\text { placebo group }\end{array}$ \\
\hline $\begin{array}{l}\text { Smyth et al. }{ }^{[31]} \\
1997\end{array}$ & $\begin{array}{l}\text { United Kingdom } \\
\text { RCT (double-blind, } \\
\text { placebo) } \\
\text { Prevent cisplatin toxicity } \\
\text { in ovarian cancer }\end{array}$ & $\begin{array}{l}151 \text { (female), } 21-76 \mathrm{yr} \\
6 \text { cycles of } 100 \mathrm{mg} / \mathrm{m}^{2} \pm 3 \mathrm{~g} / \\
\mathrm{m}^{2}+\text { GSH IV over } 15 \mathrm{~min}, \\
3 \mathrm{wk}\end{array}$ & $\begin{array}{l}6 \text { courses of cisplatin, } 58 \% \text { GSH v. } 39 \% \\
\text { control } \\
\text { Improved creatinine, GSH } 74 \% \text { v. } 62 \% \\
\text { ( } p=0.006) \\
\text { GSH improved depression, emesis, } \\
\text { neurotoxicity, hair loss, shortness of breath }\end{array}$ & None reported \\
\hline
\end{tabular}


Table 1. (continued) Current list of human clinical trials associated with GSH

\begin{tabular}{|c|c|c|c|c|}
\hline Reference & $\begin{array}{l}\text { Country of origin } \\
\text { Study type } \\
\text { Indication }\end{array}$ & $\begin{array}{l}\text { Subjects, } n \text { (sex), age } \\
\text { GSH dose and duration } \\
\text { Study duration }\end{array}$ & Outcomes & Adverse events \\
\hline $\begin{array}{l}\text { Schmidinger et } \\
\text { al. }^{[32]} 2000\end{array}$ & $\begin{array}{l}\text { Austria } \\
\text { RCT (blinding, pilot) } \\
\text { GSH v. intensive } \\
\text { hydration in cisplatin } \\
\text { chemo regimen for solid } \\
\text { tumours }\end{array}$ & $\begin{array}{l}20 \\
80 \mathrm{mg} / \mathrm{m}^{2}, 4 \mathrm{wk} \\
\mathrm{GSH} 5 \mathrm{~g} \mathrm{IV}+2000 \mathrm{~mL} \\
\text { saline control }+4000 \mathrm{~mL} \\
\text { saline + forced diuresis } \\
\mathrm{NR}\end{array}$ & $\begin{array}{l}\text { Haemoglobin: GSH } 10.7 \mathrm{mg} \text { v. placebo } \\
9.5 \mathrm{mg}(p=0.039) \\
\text { White cells: GSH } 3.3 \times 10^{3} / \mathrm{mL} \text { v. } \\
\text { placebo } 2.2 \times 10^{3} / \mathrm{mL}(p=0.004) \\
\text { Platelets: GSH } 167 \times 10^{3} / \mathrm{mL} \text { v. placebo } \\
95 \times 10^{3} / \mathrm{mL}(p=0.02)\end{array}$ & None reported \\
\hline $\begin{array}{l}\text { Cascinu et al. }{ }^{[33]} \\
2002\end{array}$ & $\begin{array}{l}\text { Italy } \\
\text { RCT (double-blind, } \\
\text { placebo) } \\
\text { Prevent oxaliplatin } \\
\text { toxicity in advanced } \\
\text { colorectal cancer }\end{array}$ & $\begin{array}{l}52 \\
\mathrm{GSH} 1500 \mathrm{mg} / \mathrm{m}^{2} \mathrm{IV} \text { over } \\
15 \mathrm{~min} \text { prior to oxaliplatin } \\
12 \text { treatment cycles }\end{array}$ & $\begin{array}{l}\text { Neuropathy } \\
\text { Cycle 4: } 7 \text { GSH v. } 11 \text { placebo } \\
\text { Cycle 8: 9/21 GSH v. } 15 / 19 \text { placebo } \\
\text { Cycle 12: } 3 \text { GSH arm v. } 8 \text { placebo }\end{array}$ & None reported \\
\hline $\begin{array}{l}\text { Milla et al.. }{ }^{[34]} \\
2009\end{array}$ & $\begin{array}{l}\text { Italy } \\
\text { Oxaliplatin neurotoxicity } \\
\text { in colorectal cancer } \\
\text { treated with FOLFOX4 } \\
\text { adjuvant regimen }\end{array}$ & $\begin{array}{l}27 \\
\text { GSH } 1500 \mathrm{mg} / \mathrm{m}^{2} \mathrm{IV} \text { or } \\
\text { saline solution before } \\
\text { oxaliplatin infusion } \\
12 \text { treatment cycles }\end{array}$ & $\begin{array}{l}\text { Reduced neurotoxicity GSH v. placebo } \\
(p=0.0037)\end{array}$ & None reported \\
\hline Leal et al.., ${ }^{[35]} 2014$ & $\begin{array}{l}\text { USA } \\
\text { RCT (double-blind, placebo) } \\
\text { Prevent platinum } \\
\text { peripheral neuropathy }\end{array}$ & $\begin{array}{l}185 \\
1.5 \mathrm{~g} / \mathrm{m}^{2} \mathrm{GSH} \text { IV or placebo } \\
\text { over } 15 \mathrm{~min} \\
18 \mathrm{wk}\end{array}$ & $\begin{array}{l}\text { No statistically significant differences } \\
\text { in peripheral neurotoxicity, degree of } \\
\text { paclitaxel acute pain syndrome, time to } \\
\text { disease progression or apparent toxicities }\end{array}$ & None reported \\
\hline \multicolumn{5}{|c|}{ Neurodegenerative disorders } \\
\hline $\begin{array}{l}\text { Sechi et al.. }{ }^{[36]} \\
1996\end{array}$ & $\begin{array}{l}\text { Italy } \\
\text { Cases (uncontrolled) } \\
\text { Parkinson's disease }\end{array}$ & $\begin{array}{l}9 \\
600 \mathrm{mg} \text { GSH IV twice daily, } \\
30 \mathrm{~d}, 4 \mathrm{mo}\end{array}$ & $\begin{array}{l}42 \% \text { decline in disability, therapeutic } \\
\text { effect lasted } 2-4 \text { mo }\end{array}$ & None reported \\
\hline $\begin{array}{l}\text { Hauser et al., }{ }^{[37]} \\
2009\end{array}$ & $\begin{array}{l}\text { USA } \\
\text { RCT, pilot trial (placebo) } \\
\text { Safety and preliminary } \\
\text { efficacy in Parkinson's } \\
\text { disease }\end{array}$ & $\begin{array}{l}21 \\
1400 \mathrm{mg} \text { GSH IV or } \\
\text { placebo } 3 \text { times a wk, } 4 \mathrm{wk} \\
3 \text { mo }\end{array}$ & $\begin{array}{l}\text { Unified Parkinson's Disease Rating Scale } \\
\text { (UPDRS) motor scores higher in GSH } \\
\text { group v. placebo }\end{array}$ & $\begin{array}{l}\text { No adverse events } \\
\text { due to GSH }\end{array}$ \\
\hline $\begin{array}{l}\text { Mischley et al. }{ }^{[38]} \\
2015\end{array}$ & $\begin{array}{l}\text { USA } \\
\text { Safety and tolerability } \\
\text { of intranasal GSH in } \\
\text { Parkinson's disease }\end{array}$ & $\begin{array}{l}30 \\
600 \mathrm{mg} / \mathrm{d} \text { of intranasal } \\
\text { GSH v. placebo (saline) in } \\
3 \text { divided daily doses } \\
3 \text { mo }\end{array}$ & All groups met tolerability criteria & $\begin{array}{l}\text { No adverse events } \\
\text { due to GSH }\end{array}$ \\
\hline
\end{tabular}

potentially severe complications (septicaemia, infective endocarditis and transmission of blood-borne infections) of IV administration of GSH by people with no health qualifications. Recent Food and Drug Administration warnings also point to a need for increased public awareness of potential harm. ${ }^{[74]}$

\section{Conclusion}

This brief review evaluates recent clinical studies on the use of GSH. Despite widespread use of IV GSH, no clinical report was identified. Large RCTs of long-enough duration and follow-up are warranted for the safe treatment of pigmentary disorders. The psychosocial impact of systemic skin lightening is a Pandora's box best addressed by multidisciplinary teams including social scientists, psychologists and psychiatrists.

Source of funding. Funded by the South African Medical Research Council.
1. Jutley GS, Rajaratnam R, Halpern J, Salim A, Emmett C. Systematic review of randomized controlled trials on interventions for melasma: An abridged Cochrane review. J Am Acad Dermatol 2014;70(2):369-373. DOI:10.1016/j.jaad.2013.07.04

2. Maneli MH, Wiesner L, Tinguely C, et al. Combinations of potent topical steroids, mercury and hydroquinone are common in internationally manufactured skin-lightening products: A spectroscopic study. Clin Exp Dermatol 2016;41(2):196-201. DOI:10.1111/ced.12720

3. Hopkins FG. On an autoxidisable constituent of the cell. Biochem J 1921;15(2):286.

4. Quastel JH, Stewart CP, Tunnicliffe HE. On glutathione. IV. Constitution. Biochem J 1923;17(4-5):586592.

5. Aoyama K, Suh SW, Hamby AM, et al. Neuronal glutathione deficiency and age-dependent neurodegeneration in the EAAC1 deficient mouse. Nat Neurosci 2006;9(1):119-126. DOI:10.1038/ $\mathrm{nn} 1609$

6. Aoyama K, Nakaki T. Impaired glutathione synthesis in neurodegeneration. Int J Mol Sci 2013;14(10):21021-21044. DOI:10.3390/ijms141021021

7. Schafer FQ, Buettner GR. Redox environment of the cell as viewed through the redox state of the glutathione disulfide/glutathione couple. Free Radic Biol Med 2001;30(11):1191-1212. DOI:10.1016/ glutathione disulfide/glutathione couple. Free Radic Biol Med 2001;30(11):1191-1212. DOI:10.1016/

8. Dickinson DA, Forman HJ. Glutathione in defense and signaling. Ann N Y Acad Sci 2002;973(1):488504. DOI:10.1111/j.1749-6632.2002.tb04690.x

9. Townsend DM, Tew KD, Tapiero H. The importance of glutathione in human disease. Biomed Pharmacother 2003;57(3-4):145-155. DOI:10.1016/s0753-3322(03)00043-x

10. Treweeke A, Winterburn T, Mackenzie I, et al. N-acetylcysteine inhibits platelet-monocyte conjugation in patients with type 2 diabetes with depleted intraplatelet glutathione: a randomised controlled trial. Diabetologia 2012;55(11):2920-2928. DOI:10.1007/s00125-012-2685-2

11. Solmi $\mathrm{M}$, Veronese $\mathrm{N}$, Manzato $\mathrm{E}$, et al. Oxidative stress and antioxidant levels in patients with anorexia nervosa: A systematic review and exploratory meta-analysis. Int J Eat Disord 2015;48(7):826-841. DOI:10.1002/eat.22443 
12. Frustaci A, Neri M, Cesario A, et al. Oxidative stress-related biomarkers in autism: Systematic review and meta-analyses. Free Rad Biol Med 2012.52(10):2128-2141. DOI-10.1016/j freeradbiomed 2012.03.011 13. Main P, Angley MT, O'Doherty CE, Thomas P. Fenech M. The potential role of the antioxidnt and detoxification properties of glutathione in autism spectrum disorders: a systematic review and metadetoxification properties of glutathione in autism spectrum disorders: a
analysis. Nutr Metab (Lond) 2012;9(1):35. DOI:10.1186/1743-7075-9-35

14. Loui A, Raab A, Maier RF, Brätter P, Obladen M. Trace elements and antioxidant enzymes in extremely 4. Loui A, Raab A, Maier RF, Brätter P, Obladen M. Trace elements and antioxidant enzymes in extremely
low birthweight infants. I Trace Elem Med Biol 2010;24(2):111-118. DOI:10.1016/j.jtemb.2009.11.004 5. Chusiri Y, Wongpoomchai R, Kakehashi A, et al. Non-genotoxic mode of action and possible
5. threshold for hepatocarcinogenicity of kojic acid in F344 rats. Food Chem Toxicol 2011;49(2):471-476. DOI:10.1016/j.fct.2010.11.027

16. Yokozawa T, Kim YJ. Piceatannol inhibits melanogenesis by its antioxidative actions. Biol Pharm Bull 2007;30(11):2007-2011. DOI:10.1248/bpb.30.2007

17. Plonka PM, Handjiski B, Michalczyk D, Popik M, Paus R. Oral zinc sulphate causes murine hair hypopigmentation and is a potent inhibitor of eumelanogenesis in vivo. Br J Dermatol 2006;155(1):3949. DOI:10.1111/j.1365-2133.2006.07376.x

18. DeCaprio AP. The toxicology of hydroquinone - relevance to occupational and environmental exposure. Crit Rev Toxicol 1999;29(3):283-330. DOI:10.1080/10408449991349221

19. Westerhof W, Kooyers TJ. Hydroquinone and its analogues in dermatology - a potential health risk. J Cosmet Dermatol 2005;4(2):55-59. DOI:10.1111/i.1473-2165.2005.40202.x

20. Alexis AF, Blackcloud P. Natural ingredients for darker skin types: Growing options for hyperpigmentation. J Drugs Dermatol 2013;12(9 Suppl):s123-s127.

21. Villarama $\mathrm{CD}$, Maibach HI. Glutathione as a depigmenting agent: An overview. Int J Cosmet Sci 2005;27(3):147-153. DOI:10.1111/j.1467-2494.2005.00235.x

22. Hong S-Y, Gil H-W, Yang J-O, et al. Pharmacokinetics of glutathione and its metabolites in normal subjects. J Korean Med Sci 2005;20(5):721-726. DOI:10.3346/jkms.2005.20.5.721

23. Sriharsha M, Reddy KN, Darsini TP, Reddy KS. Glutathione as a whitening agent in the treatment of melanosis of the face. Int J Pharm Drug Res 2015;5(1):1-6.

24. Arjinpathana N, Asawanonda P. Glutathione as an oral whitening agent: A randomized, double-blind, placebo-controlled study. J Dermatolog Treat 2012;23(2):97-102. DOI:10.3109/09546631003801619

25. Watanabe F, Hashizume E, Chan GP, Kamimura A. Skin-whitening and skin-condition-improving effects of topical oxidized glutathione: A double-blind and placebo-controlled clinical trial in healthy women. Clin Cosmet Investig Dermatol 2014;7:267. DOI:10.2147/ccid.s68424

26. Handog EB, Datuin MSL, Singzon IA. An open-label, single-arm trial of the safety and efficacy of a novel preparation of glutathione as a skin-lightening agent in Filipino women. Int J Dermatol 2015;55(2):153-157. DOI:10.1111/ijd.12999

27. Cascinu S, Cordella L, del Ferro E, Fronzoni M, Catalano G. Neuroprotective effect of reduced glutathione on cisplatin-based chemotherapy in advanced gastric cancer: A randomized double-blind glutathione on cisplatin-based chemotherapy in advanced
placebo-controlled trial. J Clin Oncol 1995;13(1):26-32.

28. Colombo N, Bini S, Miceli D, et al. Weekly cisplatin +/-glutathione in relapsed ovarian carcinoma. Int J Gynecol Cancer 1995;5(2):81-86. DOI:10.1046/j.1525-1438.1995.05020081.x

29. Parnis FX, Coleman RE, Harper PG, et al. A randomised double-blind placebo controlled clinical trial assessing the tolerability and efficacy of glutathione as an adjuvant to escalating doses of cisplatin in the treatment of advanced ovarian cancer. Eur J Cancer 1995;31A(10):1721. DOI:10.1016/09598049(95)00310-F

30. Bogliun G, Marzorati L, Cavaletti G, Frattola L. Evaluation by somatosensory evoked potentials of the neurotoxicity of cisplatin alone or in combination with glutathione. Ital J Neurol Sci 1992;13(8):643647. DOI:10.1007/BF02334967

31. Smyth JF, Bowman A, Perren T, et al. Glutathione reduces the toxicity and improves quality of life of women diagnosed with ovarian cancer treated with cisplatin: Results of a double-blind, randomised trial. Ann Oncol 1997:8(6):569-573.

32. Schmidinger M, Budinsky AC, Wenzel C, et al. Glutathione in the prevention of cisplatin induced toxicities. A prospectively randomized pilot trial in patients with head and neck cancer and non small toxicities. A prospectively randomized pilot trial in patients with
cell lung cancer. Wien Klin Wochenschr 2000;112(14):617-623.

33. Cascinu S, Catalano V, Cordella L, et al. Neuroprotective effect of reduced glutathione on oxaliplatinbased chemotherapy in advanced colorectal cancer: A randomized, double-blind, placebo-controlled
bated based chemotherapy in advanced colorectal cancer: A randomized, double
trial. J Clin Oncol 2002;20(16):3478-3483. DOI:10.1200/JCO.2002.07.061

trial. J Clin Oncol 2002;20(16):3478-3483. DOI:10.1200//CO.2002.07.061
34. Milla P, Airoldi M, Weber G, Drescher A, Jaehde U, Cattel L. Administration of reduced glutathione in FOLFOX4 adjuvant treatment for colorectal cancer: Effect on oxaliplatin pharmacokinetics, $\mathrm{Pt}$ DNA adduct formation, and neurotoxicity. Anticancer Drugs 2009;20(5):396-402. DOI:10.1097/ CAD.0b013e32832a2dc1.

35. Leal AD, Qin R, Atherton PJ, et al., Alliance for Clinical Trials in Oncology. North Central Cancer Treatment Group/Alliance trial N08CA - the use of glutathione for prevention of paclitaxel/ carboplatin-induced peripheral neuropathy: A phase 3 randomized, double-blind, placebo-controlled study. Cancer 2014;120(12):1890-1897. DOI:10.1002/cncr.28654

36. Sechi G, Deledda MG, Bua G, et al. Reduced intravenous glutathione in the treatment of early Parkinson's disease. Prog Neuropsychopharmacol Biol Psychiatry 1996;20(7):1159-1170. DOI:10.1016/ S0278-5846(96)00103-0

37. Hauser RA, Lyons KE, McClain T, Carter S, Perlmutter D. Randomized, double-blind, pilot evaluation of intravenous glutathione in Parkinson's disease. Mov Disord 2009;24(7):979-83. DOI:10.1002/
ond mds.22401.

38. Mischley LK, Leverenz JB, Lau RC, et al. A randomized, double-blind phase I/IIa study of intranasal glutathione in Parkinson's disease. Mov Disord 2015;30(12):1696-1701. DOI:10.1002/mds.26351

39. Villafuerte G, Miguel-Puga A, Murillo Rodríguez E, et al. Sleep deprivation and oxidative stress in animal models: A systematic review. Oxid Med Cell Longev 2015;2015:1-15. DOI:10.1155/2015/234952

40. Durg S, Dhadde SB, Vandal R, Shivakumar BS, Charan CS. Withania somnifera (Ashwagandha) in neurobehavioural disorders induced by brain oxidative stress in rodents: A systematic review and meta-analysis. J Pharm Pharmacol 2015;67(7):879-899. DOI:10.1111/jphp.12398

41. Wang L, Hong G, Li D, Chen X, Han W, Lu Z. [The efficacy of traditional Chinese medicin in animal model of lung injury induced by paraquat: A meta-analysis]. Zhonghua Wei Zhong Bing Ji Jiu Yi Xue 2014;26(6):399-404

42. Song Q-B, Wang Q, Hu W-G. A systemic review of glutathione S-transferase P1 Ile105Val polymorphism and colorectal cancer risk. Chin J Cancer Res 2014;26(3):255. DOI:10.3978/.jissn.10009604.2014.06.01

43. Ye Z, Song H. Glutathione s-transferase polymorphisms (GSTM1, GSTP1 and GSTT1) and the risk of acute leukaemia: A systematic review and meta-analysis. Eur J Cancer 2005;41(7):980-989. DOI:10.1016/j.jejca.2005.01.014
44. Xiao Q, Deng D, Li H, et al. GSTT1 and GSTM1 polymorphisms predict treatment outcome for acute myeloid leukemia: A systematic review and meta-analysis. Ann Hematol 2014;93(8):1381-1390. DOI:10.1007/s00277-014-2050-z

45. Yang H, Yang S, Liu J, Shao F, Wang H, Wang Y. The association of GSTM1 deletion polymorphism with lung cancer risk in Chinese population: Evidence from an updated meta-analysis. Sci Rep 2015;5:9392. DOI: $10.1038 /$ srep09392

46. Shi X, Zhou S, Wang Z, Zhou Z, Wang Z. CYP1A1 and GSTM1 polymorphisms and lung cancer risk in Chinese populations: A meta-analysis. Lung Cancer 2008;59(2):155-163. DOI:10.1016/j. lungcan.2007.08.004

47. Wu K, Wang X, Xie Z, Liu Z, Lu Y. Glutathione S-transferase P1 gene polymorphism and bladder cance susceptibility: An updated analysis. Mol Biol Rep 2013;40(1):687-695. DOI:10.1007/s11033-012-2109-7 8. Wang H, Zhou Y, Zhuang W, et al. Glutathione S-transferase M1 null genotype associated with gastric cancer among Asians. Dig Dis Sci 2010;55(7):1824-1830. DOI:10.1007/s10620-009-0971-5

49. Yoon J, Hyun M-H, Yang J-P, Park M-J, Park S. Ethnic differences in the association of the glutathion S-transferase T1 (GSTT1) null genotype and risk of gastric carcinoma: A systematic review and metaanalysis. Mol Biol Rep 2014;41(6):3867-3879. DOI:10.1007/s11033-014-3254-y

50. Yu Z, Li Z, Cai B, et al. Association between the GSTP1 Ile105Val polymorphism and prostate cancer risk: A systematic review and meta-analysis. Tumor Biol 2013;34(3):1855-1863. DOI:10.1007/s13277-013-0727-x

51. Cai Q, Wu T, Zhang W, et al. Genetic polymorphisms in glutathione S-transferases P1 (GSTP1) Ile105Val and prostate cancer risk: A systematic review and meta-analysis. Tumor Biol 2013;34(6):39133922. DOI:10.1007/s13277-013-0979-5

52. Cai Q, Wang Z, Zhang W, et al. Association between glutathione S-transferases M1 and T1 gene polymorphisms and prostate cancer risk: A systematic review and meta-analysis. Tumour Biol 2014:35(1):247-256. DOI:10.1007/s13277-013-1030-6

53. Liu D, Liu Y, Ran L, Shang H, Li D. GSTT1 and GSTM1 polymorphisms and prostate cancer risk in Asians: A systematic review and meta-analysis. Tumor Biol 2013;34(5):2539-2544. DOI:10.1007 s13277-013-0778-z

54. Lai R, Crevier L, Thabane L. Genetic polymorphisms of glutathione S-transferases and the risk of adult brain tumors: A meta-analysis. Cancer Epidemiol Biomarkers Prev 2005;14(7):1784-1790. DOI:10.1158/1055-9965.epi-05-0105

55. Peng H, He Q, Zhu J, Peng C. Effect of GSTM1 polymorphism on risks of basal cell carcinoma and squamous cell carcinoma: A meta-analysis. Tumour Biol 2012;34(2):675-681. DOI:10.1007/s13277012-0595-9

56. Ge B, Song Y, Zhang Y, Liu X, Wen Y, Guo X. Glutathione S-transferase M1 (GSTM1) and T1 (GSTT1) null polymorphisms and the risk of hypertension: A meta-analysis. PloS One 2015;10(3):e0118897. DOI:10.1371/journal.pone.0118897

57. Eslami S, Sahebkar A. Glutathione-S-transferase M1 and T1 null genotypes are associated with hypertension risk: A systematic review and meta-analysis of 12 studies. Curr Hypertens Rep 2014;16(6):1-9. DOI:10.1007/s1 1906-014-0432-1

58. Minelli C, Granell R, Newson R, et al. Glutathione-S-transferase genes and asthma phenotypes: A Human Genome Epidemiology (HuGE) systematic review and meta-analysis including unpublished data. Int J Epidemiol 2009:39(2):539-562. DOI:10.1093/ije/dyp337

59. Piacentini S, Polimanti R, Simonelli I, et al. Glutathione S-transferase polymorphisms, asthm susceptibility and confounding variables: A meta-analysis. Mol Biol Rep 2013;40(4):3299-3313. DOI:10.1007/s11033-012-2405-2

60. Liao R-F, Ye M-I, Liu C-Y, Ye D-Q. An updated meta-analysis: Risk conferred by glutathione S-transferases (GSTM1 and GSTT1) polymorphisms to age-related cataract. J Ophthalmo 2015;2015:1-10. DOI:10.1155/2015/103950

61. Dahabreh IJ, Giannouli S, Gota V, Voulgarelis M. GSTT1 and GSTM1 polymorphisms and myelodysplastic syndrome risk: A systematic review and meta-analysis. Int J Cancer 2010;126(7):17161723. DOI:10.1002/ijc.24940

62. Fang $\mathrm{M}$, Zeng $\mathrm{W}$, Huang $\mathrm{L}$, et al. Association between glutathione S-transferase $\mathrm{T} 1$ null genotype and risk of myelodysplastic syndromes: A comprehensive meta-analysis. Tumour Biol 2013;34(5):27472752. DOI: $10.1007 / \mathrm{s} 13277-013-0828-6$

63. Xie P, Liang Y, Liang G, Liu B. Association between GSTP1 Ile105Val polymorphism and glioma risk: A systematic review and meta-analysis. Tumor Biol 2014;35(1):493-499. DOI:10.1007/s13277-013-1069-4

64. Li X, Pan J, Liu Q, et al. Glutathione S-transferases gene polymorphisms and risk of male idiopathic infertility: A systematic review and meta-analysis. Mol Biol Rep 2013;40(3):2431-2438. DOI:10.1007 s11033-012-2323-3

65. Albers JW, Chaudhry V, Cavaletti G, Donehower RC. Interventions for preventing neuropathy caused by cisplatin and related compounds. Cochrane Database Syst Rev 2014, Issue 3. Art. No.: CD005228. DOI:10.1002/14651858.CD005228.pub4

66. Villarama C, Maibach H. Glutathione as a depigmenting agent: An overview. Int J Cosmet Sci 2005;27(3):147-153. DOI:10.1111/j.1467-2494.2005.00235.

67. Ebanks JP, Wickett RR, Boissy RE. Mechanisms regulating skin pigmentation: The rise and fall of complexion coloration. Int J Mol Sci 2009;10(9):4066-4087. DOI:10.3390/ijms10094066

68. Del Marmol V, Beermann F. Tyrosinase and related proteins in mammalian pigmentation. FEBS Lett 1996;381(3):165-168. DOI:10.1016/0014-5793(96)00109-3

69. Benedetto J-P, Ortonne J-P, Voulot C, Khatchadourian C, Prota G, Thivolet J. Role of thiol compound in mammalian melanin pigmentation. II. Glutathione and related enzymatic activities. J Invest Dermatol 1982;79(6):422-424. DOI:10.1111/1523-1747.ep12530631

70. Halprin KM, Ohkawara A. Glutathione and human pigmentation. Arch Dermatol 1966;94(3):355-357. DOI:10.1001/archderm.94.3.35

71. Karg E, Odh G, Wittbjer A, Rosengren E, Rorsman H. Hydrogen peroxide as an inducer of elevated tyrosinase level in melanoma cells. Invest Dermatol 1993;100(Suppl 2):209S-213S. DOI:10.1111/15231747.ep12465218

72. Dalhoff K, Ranek L, Mantoni M, Poulsen HE. Glutathione treatment of hepatocellular carcinoma. Liver 1992;12(5):341-343. DOI:10.1111/j.1600-0676.1992.tb00583.x

73. Wenczl E, van der Schans GP, Roza L, et al. (Pheo) melanin photosensitizes UVA-induced DNA damage in cultured human melanocytes. I Invest Dermatol 1998;111(4):678-682. DOI:10.1046/j.1523 1747.1998.00357.x

74. Food and Drug Administration. Injectable Skin Lightening Products: What You Should Know. http:// www.fda.gov/ForConsumers/ConsumerUpdates/ucm460788.html (accessed 5 January 2015).

Accepted 11 April 2016. 